\title{
Cloud Based Face Recognition using Machine Learning
}

\author{
Pramod R', Rakshanda D. Bellary, Riya Bharti, Sushma S.
}

Computer Science and Engineering, Nagarjuna College of Engineering and Techology, Bengaluru, Karnataka, India

\section{ABSTRACT}

\section{Article Info}

Volume 8, Issue 3

Page Number : 1003-1006

\section{Publication Issue}

May-June-2021

\section{Article History}

Accepted : 20 June 2021

Published : 30 June 2021
The main motive of this paper is to implement a system where the employees and the visitors are granted access to enter the office by recognizing their face images. And henceforth, the access is granted only when the employee or the visitor enters the correct pin into the keypad which is concerned for authentication purpose.Without any usage of the tag keys or identity card an employee can easily unlock the entrance door once his face is recognized. A Raspberry pi, a camera, a memory card and a keypad is the hardware components that is required in this system. The face recognition and the authentication carried out by the keypad is controlled by the cloud based platforms and the local based Web Services. The authentication mechanism and the face recognition provides a safe and and increased level of security which gives a protection against spoofing attacks where there is no need of carrying any tag keys or access cards.

Keywords : Face Recognition, Keypad, Cloud, Authentication, Pi Camera.

\section{INTRODUCTION}

As we all know that for a employee to enter the office today, require tag keys or identity cards. Anyone who forgets or do not have their identity cards have to ring the doorbell or seek help for that case which usually initiates a lot of disturbance and trouble for the one who is entering the office. Due to this a lot of time and focus is consumed of the employees as well as the staff memerbs nearby.

Therefore, to implement a system which not only recognizes the face of the employee who is entering but also through a security pin gets an access for the employee to enter the office by unlocking the door. There are two ways in which this project could be helpful for both the employees as well as the company. Firstly it will ease the process of any employee enntering the office. Secondly, there will be minimum amount of disturbance when someone wants to enter the office or someone forgets their tag keys. The limitations of this paper is only the fundamentals of access control system. With the help of small computer and a camera this project helps to control the lock system and also allows access for the employees. A comparison is made with the images stored in the database and with the images being 
captured which is used for identification of the employee entering.

Authetication is also very important as one enters the pin which grants access to the employee, this is basically for the security purpose which prevents the spoofing attacks. Developmemt of user interface is made via the VNC viewer which handles user interaction. Presentation of different outputs and the interaction with the users is done through the GUI(Graphical User Interface).

\section{RELATED WORKS}

We are all familiar with the emerging technologies like 2D, 3D, 2D-3D which is very common in facial recogition. The accuracy is totally dependent on the factors which are related to the pose, occlusion, or expression. Today we can see an extensive use of facial recognition technologies and also availability of personal cameras on their devices. Cloud computing plays a very important role where it stores the data and reduces the burden of storage capacity where it has a huge possibility of biometric application. However, we see a lot of face recognition devices like unlocking mobile phones, preventing retail crime, Smarter Advertising. As we all know that today to get picture of any person directly from the internet has become very easy.

This is the main reason where one should be careful and should find measures to prevent the spoofing attacks. There are being researches going on two decades for object recognition, shape matches and pattern recognition in the computer field. Here, we get to see that face recognition is the main issue.

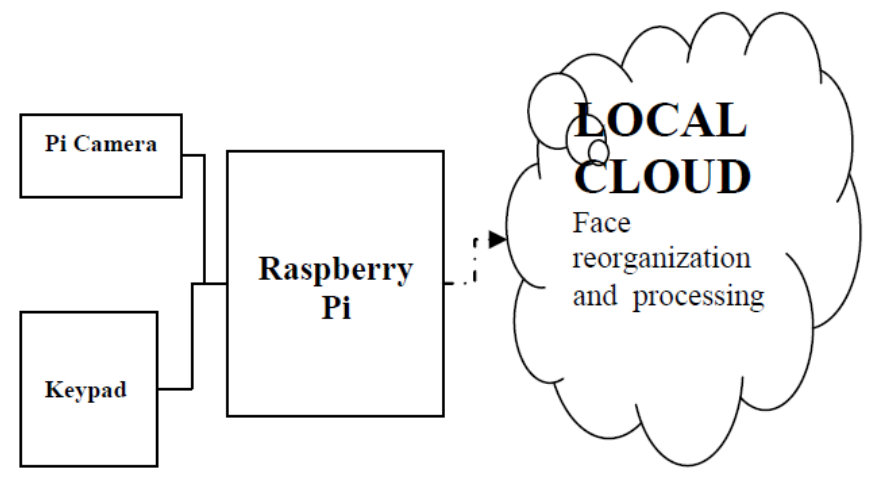

Figure 1: System Architecture

\section{PROPOSED PROTOTYPE}

The approach of this paper is to benefit the employees and staff members by permitting them into the office through face recognition and also generating a pin through keypad. Hardware specifications are a $\mathrm{Pi}$ Camera, Raspberry pi, Keypad and also a SD card. Face recognition is liked to the cloud based solutions where we store the data in the form of frame through which the comparison is made with the images clicked at the instance when the employee is entering. Entrance of the employee is depends on the recognition which has a great dominance for the access to be granted. The action of entering the office therefore, becomes a lot more simplified. This decreases the distraction for those who do not have identity card or ay tag key to get into their office. Here we note that system is split into three sections which is the Database Creation followed by the Training the dataset ad lastly ending with the Testing section. This clings on the motive of helping the employees to attend the office without being delayed.

\section{SYSTEM ANALYSIS}

The structure of the system has a Raspberry Pi which is connected to the PI camera and a SD card is inserted for storage and data purpose (Figure 1). This is further linked with the computer or any keyboard or mouse with a display device which could be anything like a TV or a monitor. Obtaining a clear 
picture, we first connect the Pi camera to the CSI interface of the Raspberry Pi board (Figure 2).

Hence we can capture the images by using Pi Camera, but before capturing the images we need to check whether the camera is enabled or not. To enable the camera, we need to configure the Raspberry Pi mode by following command : sudo raspi-config. To enable the functionality of the camera we first have to select the interfacing options followed by rebooting the Raspberry Pi and can capture the images and videos .

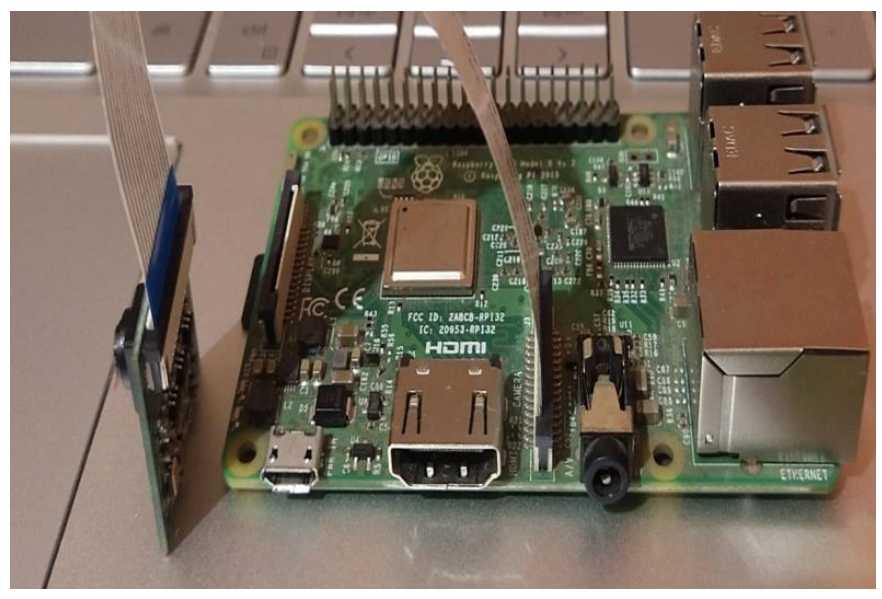

Figure 2: CSI Interface of Raspberry Pi

First and foremost, the ample amount of images which is captured may be positive or negative images that are required to train the Haar Cascade Classifier. Here we note that the positive images are the images which have clear faces and the negative images are those having no faces or blurred faces. The implementation stage describes the means through which the software and hardware are intergrated to form a complete system. Python V3 is the software component selected for programming language. Java could also be a consideration but Python offered a lot of APIs and libraries than java. Therefore, we concluded that python offers more simplified library functions and APIs for calculations and data analysis for our related work. Installation of python package for a $4^{*} 4$ and $4^{*} 3$ matrix keypad (Figure 3.) which allows all the interrupt based performance to be performed. The keypad is further devided into four columns and four rows. The keypad and the raspberry pi establishes a connection between them meanwhile the Raspberry pi accepts the information coming from the keypad. This is done because a pin is required to enter the office which is fed through the keypad only.

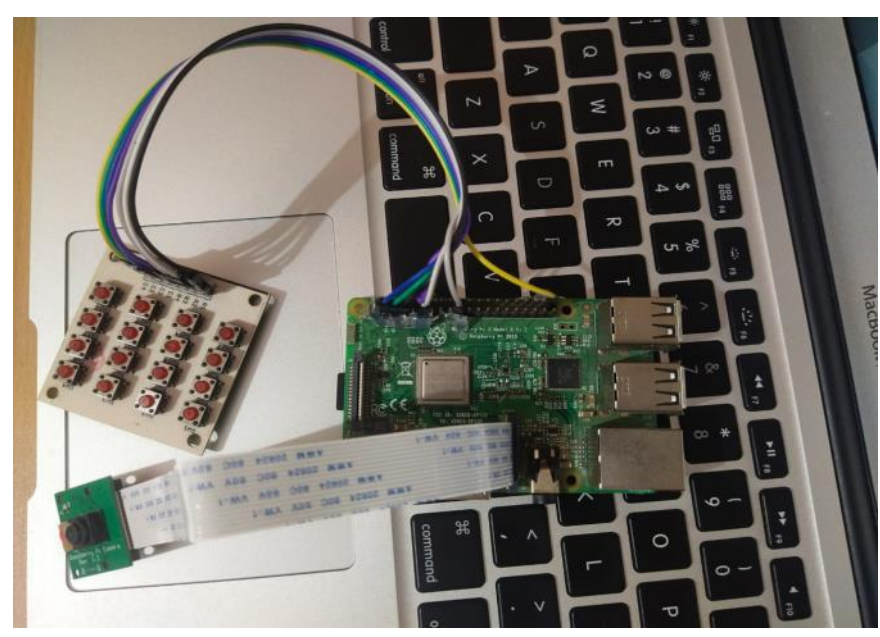

Figure 3 : Keypad interface with the Rasperry Pi model

\section{RESULTS}

The Raspberry Pi model is connected to a power cable whereas pi camera and keypad is connected to their respective slots in the system, then enabling the pi camera so that it can capture the images. Later once the software, Raspbian OS is installed the raspberry pi will be displayed which has some features same as your windows. After connecting the server we have to check the IP address and run the server. To process the image first we have to send the file to a local cloud or sever where the image is already trained which will be recognised later (Figure 4).

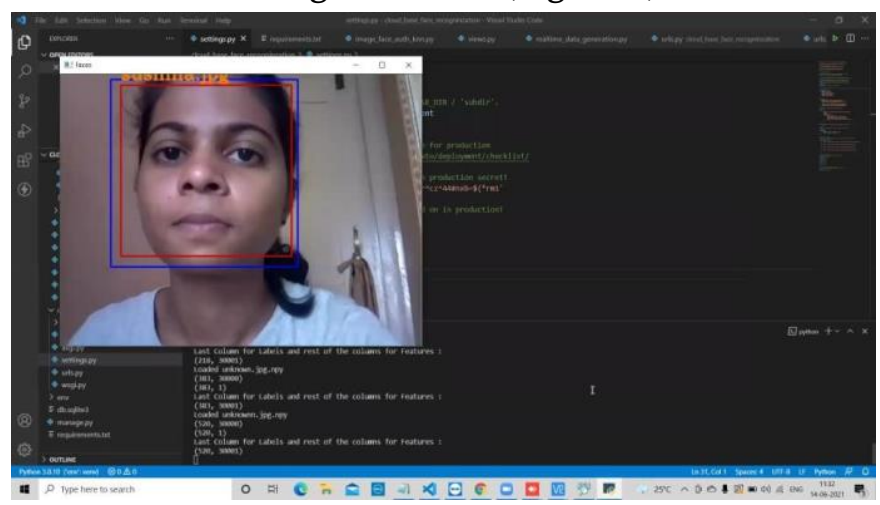

Figure 4: Image being recognized. 
Soon after the image is captured a four digit pin is asked to enter by the user which is already encrypted. User/Employee with a proper face identification and pin entered will be allowed to get the access. This system helps to prevent spoofing attacks and increase security. To carry out face recognition the cloudbased platforms local Web Services and the API service are used respectively..

\section{CONCLUSION}

In an office environment this prototype works well but as soon as there is a slower internet connection it takes time in loading as well as face recognition part also delays. Because it has a connection with cloud which works via internet so even in this case it is time consuming depending on the proper internet connection.

GUI makes the work much more easier because we see that the user interaction is done with a lot of ease. Hereafter makes the user or the employee much more convenient as it shows the instructions and information while entering the office. This process is very simplified for anyone entering the office.This works very well in an environment of work and ethics preventing from all spoofing attacks and is also beneficial for the staff members in a office.

\section{REFERENCES}

[1]. S. Singh and S. Prasad, "Techniques and challenges of face recognition: A critical review," Procedia Computer Science, vol. 143, pp. 536 - 543, 2018.

[2]. A. A. Albahdal and T. E. Boult, "Problems and promises of using the cloud and biometrics," in 11Th International Conference on Information Technology: New Generations, April 2014, pp. 293- 300 .
[3]. M. Ayad, M. Taher, and A. Salem, "Real-time mobile cloud computing: A case study in face recognition," in 28th International Conference on Advanced Information Networking and Applications Workshops, May 2014, pp. 73-78.

[4]. D. von S"ohsten and S. Murilo, "Multiple face recognition in real-time using cloud computing, emgu cv and windows azure," in 13th International Conference on Intelligent Systems Design and Applications, Dec 2013, pp. 137-140.

[5]. R. Kesava, "Cloud software as a service with iris authentication," Journal of GlobalResearch in Computer Science, 2010

\section{Cite this article as :}

Pramod R, Rakshanda D. Bellary, Riya Bharti, Sushma S, "Cloud Based Face Recognition using Machine Learning", International Journal of Scientific Research in Science and Technology (IJSRST), Online ISSN : 2395-602X, Print ISSN : 2395-6011, Volume 8 Issue 3, pp. 1003-1006, May-June 2021. Available at doi : https://doi.org/10.32628/IJSRST2183217

Journal URL : https://ijsrst.com/IJSRST2183217 